TRANSACTIONS OF THE

AMERICAN MATHEMATICAL SOCIETY

Volume 352, Number 3 , Pages 1203-1215

S 0002-9947(99)02290-4

Article electronically published on September 20, 1999

\title{
ASYMPTOTICS TOWARD THE PLANAR RAREFACTION WAVE FOR VISCOUS CONSERVATION LAW IN TWO SPACE DIMENSIONS
}

\author{
MASATAKA NISHIKAWA AND KENJI NISHIHARA
}

\begin{abstract}
This paper is concerned with the asymptotic behavior of the solution toward the planar rarefaction wave $r\left(\frac{x}{t}\right)$ connecting $u_{+}$and $u_{-}$for the scalar viscous conservation law in two space dimensions. We assume that the initial data $u_{0}(x, y)$ tends to constant states $u_{ \pm}$as $x \rightarrow \pm \infty$, respectively. Then, the convergence rate to $r\left(\frac{x}{t}\right)$ of the solution $u(t, x, y)$ is investigated without the smallness conditions of $\left|u_{+}-u_{-}\right|$and the initial disturbance. The proof is given by elementary $L^{2}$-energy method.
\end{abstract}

\section{INTRODUCTION}

We consider the Cauchy problem for the scalar viscous conservation law in two space dimensions:

$$
\begin{aligned}
u_{t}+f(u)_{x}+g(u)_{y} & =\mu \Delta u, \quad(t, x, y) \in R_{+} \times R^{2}, \\
u(0, x, y) & =u_{0}(x, y),
\end{aligned}
$$

where $f$ and $g$ are smooth functions, and $\mu$ is a positive constant. We assume that $f$ is convex, i.e.,

$$
f^{\prime \prime}(u) \geq \alpha>0 \text { for } u \in R,
$$

and that the initial data is asymptotically constant:

$$
u_{0}(x, y) \rightarrow u_{ \pm} \text {as } x \rightarrow \pm \infty \text { for any fixed } \mathrm{y} \in R,
$$

where $u_{ \pm}$are constants satisfying $u_{-}<u_{+}$. The asymptotic behavior as $t \rightarrow \infty$ of the solution is closely related to that of the Riemann problem for the corresponding hyperbolic conservation law in one space dimension:

$$
\begin{array}{r}
r_{t}+f(r)_{x}=0, \quad(t, x) \in(-1, \infty) \times R, \\
r(-1, x)=r_{0}^{R}(x) \equiv \begin{cases}u_{-} & \text {for } x<0, \\
u_{+} & \text {for } x>0\end{cases}
\end{array}
$$

Received by the editors July 8, 1996 and, in revised form, October 14, 1997.

1991 Mathematics Subject Classification. Primary 35L65, 35L67, 76L05.

Key words and phrases. Nonlinear stable, viscous conservation law, planar rarefaction wave, $L^{2}$-energy method. 
The entropy solution $r(t, x)$ of $(1.5),(1.6)$ is given by

$$
r(t, x)= \begin{cases}u_{-} & \text {for } x<f^{\prime}\left(u_{-}\right)(t+1), \\ \left(f^{\prime}\right)^{-1}\left(\frac{x}{t+1}\right) & \text { for } f^{\prime}\left(u_{-}\right)(t+1) \leq x \leq f^{\prime}\left(u_{+}\right)(t+1), \\ u_{+} & \text {for } f^{\prime}\left(u_{+}\right)(t+1)<x .\end{cases}
$$

The function $(t, x, y) \rightarrow r(t, x)$ is called the planar rarefaction wave. In a one dimensional case, the asymptotic behaviors of solutions were originally investigated by Il'in and Oleinik [3]. Harabetian [1] obtained the convergence rate toward the rarefaction wave. Hattori and Nishihara [2] showed more precise behaviors of the solution for the Burgers equation, employing the Hopf-Cole transformation. See also [5], [6], [7], [8], [10].

In a two dimensional case, Xin [9] has first investigated the stability of the planar rarefaction wave. Ito [4] has recently shown the convergence rate toward the planar rarefaction wave. In both papers, the smallness of initial disturbance is essentially assumed. In [4], the rarefaction wave is also assumed to be weak.

Our main purpose in this paper is to show that the solution $u(t, x, y)$ asymptotically behaves as $r(t, x)$ with the same rate as that in [4] without smallness conditions, which improves their results.

Denote $R_{+}^{2}=\left\{(x, y) \in R^{2} ; x>0\right\}, R_{-}^{2}=\left\{(x, y) \in R^{2} ; x<0\right\}$ and $D=\left(\frac{\partial}{\partial x}, \frac{\partial}{\partial y}\right)$. Then, our main theorem is as follows.

Theorem 1. Suppose that $u_{0}(x, y)-u_{ \pm} \in L^{2}\left(R_{ \pm}^{2}\right) \cap L^{1}\left(R_{ \pm}^{2}\right)$ and $D^{\alpha} u_{0}(x, y) \in$ $H^{1}\left(R^{2}\right),|\alpha|=1$. Then the problem (1.1),(1.2) has a unique global solution $u(t, x, y)$ satisfying

$$
\sup _{y \in R}\|u(t, \cdot, y)-r(t, \cdot)\|_{L^{2}\left(R_{x}\right)} \leq C(1+t)^{-\frac{1}{4}} \log (2+t)
$$

where $C$ is a positive constant depending on $u_{0}$.

Our plan in this paper is as follows. In the next section, we construct a smooth rarefaction wave, which is different from that in [4], and reformulate our problem. In the last two sections, we give the proofs of theorems for the reformulated problems.

\section{SMOOTH APPROXIMATION AND PRELIMINARIES}

We first introduce the function $\tilde{w}(t, x)$ as a solution to the problem:

$$
\begin{aligned}
\tilde{w}_{t}+\tilde{w} \tilde{w}_{x} & =\mu \tilde{w}_{x x}, \quad(t, x) \in(-1, \infty) \times R, \\
\tilde{w}(-1, x) & =\tilde{r}_{0}^{R}(x) \equiv f^{\prime}\left(r_{0}^{R}(x)\right) .
\end{aligned}
$$

The Hopf-Cole transformation gives the information of the properties of $\tilde{w}$. Using $\tilde{w}(t, x)$, we define "the smooth rarefaction wave" $w(t, x)$ as

$$
w(t, x)=\left.\left(f^{\prime}\right)^{-1}(\tilde{w}(t, x))\right|_{t \geq 0} .
$$

According to (1.3), $w(t, x)$ satisfies

$$
\begin{aligned}
w_{t}+f(w)_{x} & =\mu w_{x x}+\mu \frac{f^{\prime \prime \prime}(w)}{f^{\prime \prime}(w)} w_{x}^{2}, \quad(t, x) \in R_{+} \times R, \\
w(0, x) & =w_{0}(x) \equiv f^{\prime}(\tilde{w}(0, x)) .
\end{aligned}
$$

The properties of the smooth rarefaction wave $w(t, x)$ are stated in the following lemma. From now on, we denote several constants by $C$ or $c$ without confusion. 
Lemma 1 (Hattori and Nishihara [2]). The smooth rarefaction wave $w(t, x)$ given by (2.3) satisfies the following properties:

(i) $\left|w(t, x)-u_{ \pm}\right| \leq C \exp \left(-c|x|^{2}\right)$,

(ii) $w_{x}(t, x)>0$,

(iii) $\left\|w_{x}(t, \cdot)\right\|_{L^{p}(R)} \leq(1+t)^{-1+\frac{1}{p}},\left\|w_{x x}(t, \cdot)\right\|_{L^{p}(R)} \leq(1+t)^{-1}$,

(iv) $\|w(t, \cdot)-r(t, \cdot)\|_{L^{p}(R)} \leq C(1+t)^{-\frac{p-1}{2 p}}$.

Since there is a "forcing term" $\frac{f^{\prime \prime \prime}(w)}{f^{\prime \prime}(w)} w_{x}^{2}$ in the equation (2.4), we further introduce the smooth rarefaction wave $U(t, x)$ approximate to $w$, which satisfies

$$
\begin{aligned}
U_{t}+f(U)_{x} & =U_{x x}, \quad(t, x) \in R_{+} \times R, \\
U(0, x) & =U_{0}(x) \equiv f^{\prime}(\tilde{w}(0, x)) .
\end{aligned}
$$

The monotonicity in $x$ of $U(t, x)$ was obtained by Xin [9], which is important in the a priori estimates in $\S 4$.

Lemma 2 (Xin [9]). Suppose that $U_{0}(x)$ is monotonically increasing:

$$
\frac{d}{d t} U_{0}(x)>0, \quad x \in R .
$$

Then, the solution $U(t, x)$ of (2.6),(2.7) satisfies

$$
\frac{d}{d x} U(t, x)>0, \quad(t, x) \in R_{+} \times R .
$$

Thus, setting

$$
\begin{aligned}
u(t, x, y)-r(t, x) & =\{w(t, x)-r(t, x)\}+\{U(t, x)-w(t, x)\}+\{u(t, x, y)-U(t, x)\} \\
& \equiv\{w(t, x)-r(t, x)\}+v(t, x)+V(t, x, y)
\end{aligned}
$$

we have reached two reformulated problems:

$$
\begin{aligned}
v_{t}+\{f(w+v)-f(w)\}_{x} & =\mu v_{x x}-\mu \frac{f^{\prime \prime \prime}(w)}{f^{\prime \prime}(w)} w_{x}^{2}, \quad(t, x) \in R_{+} \times R, \\
v(0, x) & =U_{0}(x)-w(0, x) \equiv v_{0}(x),
\end{aligned}
$$

and

$$
\begin{gathered}
V_{t}+\{f(U+V)-f(U)\}_{x}+g(U+V)_{y}=\mu \Delta V, \quad(t, x, y) \in R_{+} \times R^{2}, \\
V(0, x, y)=u_{0}(x, y)-U_{0}(x) \equiv V_{0}(x, y) .
\end{gathered}
$$

The perturbations $v$ and $V$ satisfy the following theorems, respectively.

Theorem 2 (Decay estimate). Suppose that $v_{0} \in H^{2}(R) \cap L^{1}(R)$. Then the problem (2.10),(2.11) has a unique global solution $v(t, x)$ satisfying

$$
\begin{gathered}
v \in C^{0}\left([0, \infty) ; H^{2}(R)\right) \cap C^{0}\left([0, \infty) ; L^{1}(R)\right), \\
v_{x} \in L^{2}\left(0, T ; H^{2}(R)\right),
\end{gathered}
$$

and

$$
\|v(t, \cdot)\|_{L^{2}(R)} \leq C(1+t)^{-\frac{1}{4}} \log (2+t) .
$$


Theorem 3 (Decay estimate). Suppose that $V_{0} \in H^{2}\left(R^{2}\right) \cap L^{1}\left(R^{2}\right)$. Then, the problem (2.12),(2.13) has a unique global solution $V(t, x, y)$ satisfying

$$
V \in C^{0}\left([0, \infty) ; H^{2}(R)\right), \quad \nabla V \in L^{2}\left(0, \infty ; H^{2}(R)\right),
$$

and

$$
\sup _{y \in R}\|V(t, \cdot, y)\|_{L^{2}\left(R_{x}\right)} \leq C(1+t)^{-\frac{3}{4}} .
$$

Theorem 2, Theorem 3 and Lemma 1 (iv) yield the desired estimate (1.8). In the next two sections, we devote ourselves to the proofs of Theorems 2 and 3 , respectively.

\section{Decay estimates for the perturbation $v$}

We begin with the Cauchy problem

$$
\begin{aligned}
v_{t}+\{f(w+v)-f(w)\}_{x} & =\mu v_{x x}-\mu \frac{f^{\prime \prime \prime}(w)}{f^{\prime \prime}(w)} w_{x}^{2}, \quad(t, x) \in R_{+} \times R, \\
v(0, x) & =U(0, x)-w(0, x) \equiv v_{0}(x) .
\end{aligned}
$$

We shall show that the problem (3.1),(3.2) has a unique global solution in the solution space $X(0, \infty)$, where

$$
X_{M}(0, T)=\left\{\psi \mid \begin{array}{ll}
\psi \in C^{0}\left([0, T] ; H^{2}(R)\right), \quad \psi_{x} \in L^{2}\left(0, T ; H^{2}(R)\right) \\
\text { and } & \sup _{[0, T]}\|\psi(t, \cdot)\|_{H^{2}} \leq M
\end{array}\right\} .
$$

In what follows, we often abbreviate the domain $R$ of $H^{2}(R)$, etc.

Proposition 1 (Local existence). Suppose that $v_{0} \in H^{2}(R)$. For any $M>0$, there exists a positive constant $T_{0}$ depending on $M$ such that if $\left\|v_{0}\right\|_{H^{2}} \leq M$, then the problem (3.1), (3.2) has a unique solution $v(t, x) \in X_{2 M}\left(0, T_{0}\right)$.

Proposition 1 can be proved in a standard way. So we omit the proof. Next, we show a priori estimates of $v$.

Proposition 2 (A priori estimate). Suppose that $v$ is a solution of (3.1),(3.2) in $X_{M}(0, T)$ for positive constants $T$ and $M$. Then there exists a positive constant $C_{0}$ such that

$$
\|v(t)\|_{H^{2}}^{2}+\int_{0}^{t} \int_{R} w_{x} v^{2} d x d \tau+\int_{0}^{t}\left\|v_{x}(\tau)\right\|_{H^{2}}^{2} d \tau \leq C_{0}\left(\left\|v_{0}\right\|_{H^{2}}^{2}+1\right) .
$$

Proof. Multiplying (3.1) by $v$, we have

$$
\frac{1}{2} \frac{d}{d t} \int_{R} v^{2} d x+\int_{R} v\{f(w+v)-f(w)\}_{x} d x+\mu \int_{R} v_{x}^{2} d x=-\int_{R} v \frac{f^{\prime \prime \prime}(w)}{f^{\prime \prime}(w)} w_{x}^{2} d x .
$$

The second term of (3.4) is estimated by the following:

$$
\begin{aligned}
& \int_{R} v\{f(w+v)-f(w)\}_{x} d x=-\int_{R} v_{x}\{f(w+v)-f(w)\} d x \\
& =\int_{R}\left[-\left(\int_{w}^{w+v} f(y) d y-f(w) v\right)_{x}+\left\{f(w+v)-f(w)-f^{\prime}(w) v\right\} w_{x}\right] d x \\
& \geq \frac{\alpha}{2} \int_{R} w_{x} v^{2} d x
\end{aligned}
$$


The right hand side is estimated as follows:

$$
\left|\int_{R} v \frac{f^{\prime \prime \prime}(w)}{f^{\prime \prime}(w)} w_{x}^{2} d x\right| \leq C \int_{R} w_{x}^{2}|v| d x \leq \frac{\alpha}{4} \int_{R} w_{x}|v|^{2} d x+C \int_{R} w_{x}^{3} d x .
$$

Integrating (3.4) over $[0, \mathrm{t}]$ and using Lemma 1 (iii), we get

$$
\|v(t)\|^{2}+\int_{0}^{t} \int_{R} w_{x} v^{2} d x d \tau+\int_{0}^{t}\left\|v_{x}(\tau)\right\|^{2} d \tau \leq C_{0}\left(\left\|v_{0}\right\|^{2}+1\right)
$$

Here and later, by $\|\cdot\|$ we denote the $L^{2}$-norm in $R$ or $R^{2}$ without confusions. Next, we derive the higher order estimates. Multiplying $(3.1)$ by $\left(-v_{x x}\right)$, we have

$$
\begin{aligned}
& \frac{1}{2} \frac{d}{d t} \int_{R} v_{x}^{2} d x-\int_{R} v_{x x}\{f(w+v)-f(w)\}_{x} d x+\mu \int_{R} v_{x x}^{2} d x \\
& \quad=\mu \int_{R} v_{x x} \frac{f^{\prime \prime \prime}(w)}{f^{\prime \prime}(w)} w_{x}^{2} d x .
\end{aligned}
$$

The right-hand side is estimated as

$$
\left|\int_{R} v_{x x} \frac{f^{\prime \prime \prime}(w)}{f^{\prime \prime}(w)} w_{x}^{2} d x\right| \leq \frac{1}{4}\left\|v_{x x}\right\|^{2}+C\left\|w_{x}\right\|_{L^{4}}^{4}
$$

The second term of (3.7) is estimated as

$$
\left|\int_{R} v_{x x}\{f(w+v)-f(w)\}_{x} d x\right| \leq \frac{\mu}{4}\left\|v_{x x}\right\|^{2}+C\left\{\left\|v_{x}(t)\right\|^{2}+\int_{R} w_{x} v^{2} d x\right\} .
$$

Here, the maximum principle for a parabolic equation has been employed. Hence, we have

$$
\left\|v_{x}(t)\right\|^{2}+\int_{0}^{t}\left\|v_{x x}(\tau)\right\|^{2} d \tau \leq C_{0}\left(\left\|v_{0}\right\|_{H^{1}}^{2}+1\right) .
$$

Differentiating (3.1) twice in $x$, and multiplying it by $v_{x x}$, we have

$$
\begin{gathered}
\frac{1}{2} \frac{d}{d t}\left\|v_{x x}(t)\right\|^{2}+\int_{R} v_{x x}\{f(w+v)-f(w)\}_{x x x} d x+\mu\left\|v_{x x x}(t)\right\|^{2} \\
=-\mu \int_{R} v_{x x}\left(\frac{f^{\prime \prime \prime}(w)}{f^{\prime \prime}(w)} w_{x}^{2}\right)_{x x} d x
\end{gathered}
$$

which yields

$$
\left\|v_{x x}(t)\right\|^{2}+\int_{0}^{t}\left\|v_{x x x}(\tau)\right\|^{2} d \tau \leq C_{0}\left(\left\|v_{0}\right\|_{H^{2}}^{2}+1\right) .
$$

Thus, the proof of Proposition 3 is complete.

Combining Proposition 1 with Proposition 2, we obtain the global result.

Theorem 4 (Global existence). Suppose that $v_{0}(x) \in H^{2}(R)$. Then the problem (3.1),(3.2) has a unique global solution $v(t, x)$ satisfying

$$
v \in C^{0}\left([0, \infty) ; H^{2}(R)\right), \quad v_{x} \in L^{2}\left(0, \infty ; H^{2}(R)\right),
$$

and the estimate (3.3).

In order to obtain the decay order of $v$, we further assume that $v_{0} \in L^{1}(R)$. 
Lemma 3. Suppose that $v_{0} \in L^{1}(R) \cap H^{2}(R)$. Then the solution $v(t, x)$ also satisfies

$$
\|v(t)\|_{L^{1}} \leq\left\|v_{0}\right\|_{L^{1}}+C_{1} \log (1+t),
$$

where $C_{1}$ is a constant depending on $\left|u_{+}-u_{-}\right|$.

Proof. The $L^{1}$-estimate (3.10) of $v$ can be proved by the same method as that in [4]. So we omit the proof.

Theorem 5 (Decay estimate). Suppose that $v_{0} \in H^{2}(R) \cap L^{1}(R)$. Then, for any $0<\varepsilon<\frac{1}{2}$, the solution $v(t, x)$ of (3.1),(3.2) satisfies

$$
\begin{aligned}
& (1+t)^{k+\frac{1}{2}+\varepsilon}\left\|\partial_{x}^{k} v(t)\right\|^{2} \\
& \quad+\int_{0}^{t}(1+\tau)^{k+\frac{1}{2}+\varepsilon}\left(\int_{R} w_{x}\left|\partial_{x}^{k} v(\tau)\right|^{2} d x+\left\|\partial_{x}^{k} v_{x}(\tau)\right\|^{2}\right) d \tau \\
& \leq C I_{k}(1+t)^{\varepsilon} \rho_{k}(t), \quad k=0,1 \\
& (1+t)^{2+\varepsilon}\left\|\partial_{x}^{2} v(t)\right\|^{2} \\
& \quad+\int_{0}^{t}(1+\tau)^{2+\varepsilon}\left(\int_{R} w_{x}\left|\partial_{x}^{2} v(\tau)\right|^{2} d x+\left\|\partial_{x}^{2} v_{x}(\tau)\right\|^{2}\right) d \tau \\
& \leq C I_{2}(1+t)^{\varepsilon} \rho_{2}(t),
\end{aligned}
$$

where

$$
I_{k}=\left(\left\|v_{0}\right\|_{L^{1}}+\left\|v_{0}\right\|_{H^{k}}+1\right)^{2}, \quad k=0,1, \quad \rho_{0}=\log ^{2}(2+t), \quad \rho_{1}=\log ^{10}(2+t),
$$

and

$$
I_{2}=\left(\left\|v_{0}\right\|_{L^{1}}+\left\|v_{0}\right\|_{H^{2}}+1\right)^{\frac{70}{3}}, \quad \rho_{2}=\log ^{6}(2+t) .
$$

Remark. The estimate (3.11) with $k=0$ shows (2.14) in Theorem 2.

Proof. The proof is similar to one in Ito [4]. However, the smooth rarefaction wave $w(t, x)$ in [4] is different from ours and its estimates are done for the linearized equation around $w(t, x)$. Hence, we give the outline of the proof.

First, we show (3.11) with $k=0$. From (3.4) and Lemma 1 (iii), we have

$$
\frac{d}{d t}\|v(t)\|^{2}+\int_{R} w_{x} v^{2} d x+\left\|v_{x}(t)\right\|^{2} \leq C(1+t)^{-2} .
$$

Multiplying (3.13) by $(1+t)^{\frac{1}{2}+\varepsilon}$, we have

$$
\begin{gathered}
\frac{d}{d t}\left\{(1+t)^{\frac{1}{2}+\varepsilon}\|v(t)\|^{2}\right\}+(1+t)^{\frac{1}{2}+\varepsilon}\left(\int_{R} w_{x} v^{2} d x+\left\|v_{x}(t)\right\|^{2}\right) \\
\leq C\left\{(1+t)^{-\frac{1}{2}+\varepsilon}\|v(t)\|^{2}+(1+t)^{-\frac{3}{2}+\varepsilon}\right\} .
\end{gathered}
$$

By the Gagliardo-Nirenberg inequality

$$
\|v(t)\|^{2} \leq C\|v(t)\|_{L^{1}(R)}^{\frac{4}{3}}\left\|v_{x}(t)\right\|^{\frac{2}{3}}
$$


we obtain

$$
\begin{aligned}
& \frac{d}{d t}\left\{(1+t)^{\frac{1}{2}+\varepsilon}\|v(t)\|^{2}\right\}+(1+t)^{\frac{1}{2}+\varepsilon}\left(\int_{R} w_{x} v^{2} d x+\left\|v_{x}(t)\right\|^{2}\right) \\
& \leq C\left\{(1+t)^{-\frac{1}{2}+\varepsilon}\|v(t)\|_{L^{1}}^{\frac{4}{3}}\left\|v_{x}(t)\right\|^{\frac{2}{3}}+(1+t)^{-\frac{3}{2}+\varepsilon}\right\} \\
& \leq \frac{1}{2}(1+t)^{\frac{1}{2}+\varepsilon}\left\|v_{x}(t)\right\|^{2}+C\left\{(1+t)^{-1+\varepsilon}\|v(t)\|_{L^{1}}^{2}+(1+t)^{-\frac{3}{2}+\varepsilon}\right\} \\
& \leq \frac{1}{2}(1+t)^{\frac{1}{2}+\varepsilon}\left\|v_{x}(t)\right\|^{2}+C\left\{(1+t)^{-1+\varepsilon}\left(\left\|v_{0}\right\|_{L^{1}}^{2}+C_{1} \log ^{2}(1+t)\right)+(1+t)^{-\frac{3}{2}+\varepsilon}\right\}
\end{aligned}
$$

that is,

$$
\begin{aligned}
& \frac{d}{d t}\left\{(1+t)^{\frac{1}{2}+\varepsilon}\|v(t)\|^{2}\right\}+(1+t)^{\frac{1}{2}+\varepsilon}\left(\int_{R} w_{x} v^{2} d x+\left\|v_{x}(t)\right\|^{2}\right) \\
& \leq C\left\{(1+t)^{-1+\varepsilon}\left(\left\|v_{0}\right\|_{L^{1}}^{2}+C_{1} \log ^{2}(1+t)\right)+(1+t)^{-\frac{3}{2}+\varepsilon}\right\} .
\end{aligned}
$$

Integrating (3.16) over $[0, t]$ in $t$, we obtain $(3.11)$ with $k=0$.

Next, we derive (3.11) with $k=1$. From (3.7), we have

$$
\frac{1}{2} \frac{d}{d t}\left\|v_{x}(t)\right\|^{2}-\int_{R} v_{x x}\{f(w+v)-f(w)\}_{x} d x+\mu\left\|v_{x x}(t)\right\|^{2} \leq C(1+t)^{-3} .
$$

Here

$$
\begin{aligned}
& -\int_{R} v_{x x}\{f(w+v)-f(w)\}_{x} d x \\
& =\frac{1}{2} \int_{R} f^{\prime \prime}(w+v) w_{x} v_{x}^{2} d x+\int_{R}\left[\frac{1}{2} v_{x}^{3}-v_{x x}\left\{f^{\prime}(w+v)-f^{\prime}(w)\right\} w_{x}\right] d x .
\end{aligned}
$$

Hence, due to (1.3), we have

$$
\begin{aligned}
& \frac{d}{d t}\left\|v_{x}(t)\right\|^{2}+\alpha \int_{R} w_{x} v_{x}^{2} d x+\left\|v_{x x}(t)\right\|^{2} \\
& \leq C\left\{\int_{R}\left|v_{x x}\|v\| w_{x}\right| d x+\int_{R}\left|v_{x}\right|^{3} d x+(1+t)^{-3}\right\} \\
& \leq \frac{1}{2}\left\|v_{x x}(t)\right\|^{2}+C\left\{\int_{R} w_{x}^{2} v^{2} d x+\left\|v_{x}(t)\right\|_{L^{3}}^{3}+(1+t)^{-3}\right\} .
\end{aligned}
$$

Multiplying (3.18) by $(1+t)^{\frac{3}{2}+\varepsilon}$, we have

$$
\begin{array}{r}
\frac{d}{d t}\left\{(1+t)^{\frac{3}{2}+\varepsilon}\left\|v_{x}(t)\right\|^{2}\right\}+\alpha(1+t)^{\frac{3}{2}+\varepsilon} \int_{R} w_{x} v_{x}^{2} d x+(1+t)^{\frac{3}{2}+\varepsilon}\left\|v_{x x}(t)\right\|^{2} \\
\leq C\left\{(1+t)^{\frac{1}{2}+\varepsilon}\left\|v_{x}(t)\right\|^{2}+(1+t)^{\frac{3}{2}+\varepsilon} \int_{R} w_{x}^{2} v^{2} d x\right. \\
\left.+(1+t)^{\frac{3}{2}+\varepsilon}\left\|v_{x}(t)\right\|_{L^{3}}^{3}+(1+t)^{-\frac{3}{2}+\varepsilon}\right\} .
\end{array}
$$

Noting that

$$
\begin{aligned}
(1+t)^{\frac{3}{2}+\varepsilon} \int_{R} w_{x}^{2} v^{2} d x & \leq(1+t)^{\frac{3}{2}+\varepsilon}\left\|w_{x}(t)\right\|_{L^{\infty}} \int_{R} w_{x} v^{2} d x \\
& \leq C(1+t)^{\frac{1}{2}+\varepsilon} \int_{R} w_{x} v^{2} d x
\end{aligned}
$$


and making use of (3.11) with $k=0$ and the Gagliardo-Nirenberg inequality

$$
\left\|v_{x}(t)\right\|_{L^{3}(R)}^{3} \leq C\left\|v_{x x}(t)\right\|_{L^{2}(R)}^{\frac{7}{4}}\|v(t)\|_{L^{2}(R)}^{\frac{5}{4}},
$$

we obtain

$$
\begin{aligned}
& (1+t)^{\frac{3}{2}+\varepsilon}\left\|v_{x}^{2}(t)\right\|^{2}+\int_{0}^{t}(1+\tau)^{\frac{3}{2}+\varepsilon}\left(\alpha \int_{R} w_{x} v_{x}^{2} d x+\left\|v_{x x}(\tau)\right\|^{2}\right) d \tau \\
& \leq C\left\{I_{0}(1+t)^{\varepsilon} \rho_{0}+\int_{0}^{t}(1+\tau)^{\frac{3}{2}+\varepsilon}\|v(\tau)\|_{L^{2}(R)}^{10} d \tau\right\} \\
& \leq C\left\{I_{0}(1+t)^{\varepsilon} \rho_{0}+\int_{0}^{t}(1+\tau)^{\frac{3}{2}+\varepsilon}\left(I_{0}(1+\tau)^{-\frac{1}{2}} \rho_{0}\right)^{5} d \tau\right\},
\end{aligned}
$$

which yields (3.11) with $k=1$. Finally, multiply (3.9) by $(1+t)^{2+\varepsilon}$ and use (3.11). After several calculations, we can obtain the desired estimate (3.12). Though the details are omitted, we cannot multiply $(3.9)$ by $(1+t)^{\frac{5}{2}+\varepsilon}$ in our method. Because we have the decay order $\left\|w_{x x}(t)\right\|^{2}=O\left(t^{-2}\right)$, not $O\left(t^{-\frac{5}{2}}\right)$ (cf. Ito [4]).

Thus the proof is complete.

\section{Decay estimates for the Perturbation $V$}

In this section, we consider the Cauchy problem in two space dimension:

$$
\begin{gathered}
V_{t}+\{f(U+V)-f(U)\}_{x}+g(U+V)_{y}=\Delta V, \\
V(0, x, y)=V_{0}(x, y) \equiv u_{0}(x, y)-U_{0}(x) .
\end{gathered}
$$

The solution space is

$$
\tilde{X}_{M}(0, T)=\left\{\psi \mid \begin{array}{cc}
\psi \in C^{0}\left([0, T] ; H^{2}\left(R^{2}\right)\right), \quad \nabla \psi \in L^{2}\left(0, T ; H^{2}\left(R^{2}\right)\right) \\
\text { and } & \sup _{[0, T]}\|\psi(t, \cdot, \cdot)\|_{H^{2}} \leq M
\end{array}\right\},
$$

with $T>0$. Then we have

Proposition 3 (Local existence). Suppose that $V_{0} \in H^{2}\left(R^{2}\right)$. For any $M>0$, there exists a positive constant $T_{0}$ depending on $M$ such that if $\left\|V_{0}\right\|_{H^{2}} \leq M$, then the problem (4.1), (4.2) has a unique solution $V(t, x, y) \in \tilde{X}_{2 M}\left(0, T_{0}\right)$.

Proposition 3 can be proved in a standard way. So we omit the proof. Next, we show a priori estimates of $V$.

Proposition 4 (A priori estimate). Suppose that $V$ is a solution of (4.1),(4.2) in $\tilde{X}_{M}(0, T)$ for positive constants $T$ and $M$. Then there exists a positive constant $C_{1}$ depending on $V_{0}$ such that

$$
\|V(t)\|_{H^{2}}^{2}+\int_{0}^{t} \int_{R^{2}} U_{x} V^{2} d x d y d \tau+\int_{0}^{t}\|\nabla V(\tau)\|_{H^{2}}^{2} d \tau \leq C_{1}\left\|V_{0}\right\|_{H^{2}}^{2} .
$$

Proof. Multiplying (4.1) by $V$ and integrating the resultant equation over $R^{2}$, we have

$$
\begin{aligned}
\frac{1}{2} \frac{d}{d t}\|V(t)\|^{2} & +\int_{R^{2}} V\{f(U+V)-f(U)\}_{x} d x d y \\
& +\int_{R^{2}} V g(U+V)_{y} d x d y+\mu\|\nabla V(t)\|^{2}=0 .
\end{aligned}
$$


The second and third terms are, respectively, estimated as follows:

$$
\begin{gathered}
\int_{R^{2}} V\{f(U+V)-f(U)\}_{x} d x d y=-\int_{R^{2}} V_{x}\{f(U+V)-f(U)\} d x d y \\
=\int_{R^{2}}\left[-\left(\int_{U}^{U+V} f(y) d y-f(U) V\right)_{x}\right. \\
\left.\quad+\left\{f(U+V)-f(U)-f^{\prime}(U) V\right\} U_{x}\right] d x d y \\
\geq \frac{\alpha}{2} \int_{R^{2}} U_{x} V^{2} d x d y .
\end{gathered}
$$

Since $U$ is independent of $y$,

$$
\begin{aligned}
\int_{R^{2}} V g(U+V)_{y} d x d y & =-\int_{R^{2}} V_{y} g(U+V) d x d y \\
& =-\int_{R^{2}} \partial_{y}\left(\int_{U}^{U+V} g(\xi) d \xi\right) d x d y=0 .
\end{aligned}
$$

Using (4.5) and (4.6), we have the basic estimate

$$
\|V(t)\|^{2}+\int_{0}^{t} \int_{R^{2}} U_{x} V^{2} d x d y d \tau+\int_{0}^{t}\|\nabla V(\tau)\|^{2} d \tau \leq C\left\|V_{0}\right\|^{2} .
$$

The estimates of the derivatives in $x, y$ of $V$ can be obtained similarly to those in Proposition 2. We omit the details.

The combination of Propositions 3 and 4 gives the global result.

Theorem 6 (Global existence). Suppose that $V_{0}(x) \in H^{2}\left(R^{2}\right)$. Then the problem (4.1),(4.2) has a unique global solution $V(t, x, y)$ satisfying

$$
V \in C^{0}\left([0, \infty) ; H^{2}(R)\right), \quad \nabla V \in L^{2}\left(0, \infty ; H^{2}(R)\right) .
$$

and the estimate (4.3).

We now show the decay estimates on $V$. As in Lemma 3 , the following $L^{1}$ estimate plays an important roll.

Lemma 4 (Ito [4]). Suppose further, in Theorem 6, that $V_{0} \in L^{1}\left(R^{2}\right)$. Then the solution $V(t, x, y)$ also satisfies

$$
\|V(t)\|_{L^{1}\left(R^{2}\right)} \leq\left\|V_{0}\right\|_{L^{1}\left(R^{2}\right)} .
$$

Applying Lemma 4, we have the following theorem.

Theorem 7 (Decay estimate). Suppose that $V_{0}(x, y) \in H^{2}\left(R^{2}\right) \cap L^{1}\left(R^{2}\right)$ and let $V(t, x, y)$ be the solution of (4.1),(4.2). Then, for any $\varepsilon>0$, there exists a constant $C>0$ such that the following decay estimates hold:

$$
\begin{aligned}
(1+t)^{1+\varepsilon}\|V(t)\|^{2} & \\
& +\int_{0}^{t}(1+\tau)^{1+\varepsilon}\left(\int_{R^{2}} U_{x}|V(\tau)|^{2} d x d y+\|\nabla V(\tau)\|^{2}\right) d \tau \\
\leq & C(1+t)^{\varepsilon}\left(\left\|V_{0}\right\|_{L^{1}}+\left\|V_{0}\right\|\right)^{2},
\end{aligned}
$$


1212 MASATAKA NISHIKAWA AND KENJI NISHIHARA

$$
\begin{aligned}
&(1+t)^{\frac{15}{8}+\varepsilon}\left\|V_{x}(t)\right\|^{2} \\
&+\int_{0}^{t}(1+\tau)^{\frac{15}{8}+\varepsilon}\left(\int_{R^{2}} U_{x}\left|V_{x}(\tau)\right|^{2} d x d y+\left\|\nabla V_{x}(\tau)\right\|^{2}\right) d \tau \\
& \leq C(1+t)^{\varepsilon} \log ^{4}(2+t)\left(\left\|V_{0}\right\|_{L^{1}}+\left\|V_{0}\right\|_{H^{1}}\right)^{2}, \\
&(1+t)^{2+\varepsilon}\left\|V_{y}(t)\right\|^{2} \\
&+\int_{0}^{t}(1+\tau)^{2+\varepsilon}\left(\int_{R^{2}} U_{x}\left|V_{y}(\tau)\right|^{2} d x d y+\left\|\nabla V_{y}(\tau)\right\|^{2}\right) d \tau \\
& \leq C(1+t)^{\varepsilon}\left(\left\|V_{0}\right\|_{L^{1}}+\left\|V_{0}\right\|_{H^{1}}\right)^{2}, \\
&(1+t)^{\frac{39}{16}+\varepsilon}\left\|V_{x x}(t)\right\|^{2} \\
&+ \int_{0}^{t}(1+\tau)^{\frac{39}{16}+\varepsilon}\left(\int_{R^{2}} U_{x}\left|V_{x x}(\tau)\right|^{2} d x d y+\left\|\nabla V_{x x}(\tau)\right\|^{2}\right) d \tau \\
& \leq C(1+t)^{\varepsilon} \log ^{8}(2+t)\left(\left\|V_{0}\right\|_{L^{1}}+\left\|V_{0}\right\|_{H^{2}}\right)^{2}, \\
&(1+t)^{\frac{23}{8}}+\varepsilon\left\|V_{x y}(t)\right\|^{2} \\
&+ \int_{0}^{t}(1+\tau)^{\frac{23}{8}+\varepsilon}\left(\int_{R^{2}} U_{x}\left|V_{x y}(\tau)\right|^{2} d x d y+\left\|\nabla V_{x y}(\tau)\right\|^{2}\right) d \tau \\
& \leq C(1+t)^{\varepsilon} \log ^{8}(2+t)\left(\left\|V_{0}\right\|_{L^{1}}+\left\|V_{0}\right\|_{H^{2}}\right)^{2}, \\
&(1+t)^{3+\varepsilon}\left\|V_{y y}(t)\right\|^{2} \\
&+\int_{0}^{t}(1+\tau)^{3+\varepsilon}\left(\int_{R^{2}} U_{x}\left|V_{y y}(\tau)\right|^{2} d x d y+\left\|\nabla V_{y y}(\tau)\right\|^{2}\right) d \tau \\
& \leq C(1+t)^{\varepsilon}\left(\left\|V_{0}\right\|_{L^{1}}+\left\|V_{0}\right\|_{H^{2}}\right)^{2} .
\end{aligned}
$$

Remark. From (4.9) and (4.11), the estimate (2.15) in Theorem 3 is obtained as

$$
\begin{aligned}
\sup _{y}\|V(t, \cdot, y)\|^{2} & \leq C\|V(t, \cdot, \cdot)\|\left\|V_{y}(t, \cdot, \cdot)\right\| \\
& \leq C(1+t)^{-\frac{1}{2}-1}=C(1+t)^{-\frac{3}{2}} .
\end{aligned}
$$

Proof. From (4.4)-(4.6), we get

$$
\frac{1}{2} \frac{d}{d t}\|V(t)\|^{2}+\frac{1}{2} \alpha \int_{R^{2}} U_{x} V^{2} d x d y+\mu\|\nabla V(t)\|^{2} \leq 0 .
$$

Multiplying (4.15) by $2(1+t)^{1+\varepsilon}$, we have

$$
\begin{gathered}
\frac{d}{d t}\left\{(1+t)^{1+\varepsilon}\|V(t)\|^{2}\right\}+(1+t)^{1+\varepsilon}\left(\alpha \int_{R^{2}} U_{x} V^{2} d x d y+2\|\nabla V(t)\|^{2}\right) \\
\leq(1+\varepsilon)(1+t)^{\varepsilon}\|V(t)\|^{2} .
\end{gathered}
$$

By the Gagliardo-Nirenberg inequality

$$
\|V(t)\|_{L^{2}\left(R^{2}\right)}^{2} \leq C\|V(t)\|_{L^{1}\left(R^{2}\right)}\|\nabla V(t)\|_{L^{2}\left(R^{2}\right)},
$$


we obtain

$$
\begin{aligned}
\frac{d}{d t}\left\{(1+t)^{1+\varepsilon}\|V(t)\|^{2}\right\}+(1+t)^{1+\varepsilon}\left(\alpha \int_{R^{2}} U_{x} V^{2} d x d y+2\|\nabla V(t)\|^{2}\right) \\
\quad \leq C(1+t)^{\frac{1+\varepsilon}{2}}\|\nabla V(t)\|(1+t)^{\frac{\varepsilon-1}{2}}\|V(t)\|_{L^{1}\left(R^{2}\right)} \\
\quad \leq(1+t)^{1+\varepsilon}\|\nabla V(t)\|^{2}+C(1+t)^{\varepsilon-1}\|V(t)\|_{L^{1}\left(R^{2}\right)}^{2} \\
\leq(1+t)^{1+\varepsilon}\|\nabla V(t)\|^{2}+C(1+t)^{\varepsilon-1}\left\|V_{0}\right\|_{L^{1}\left(R^{2}\right)}^{2}
\end{aligned}
$$

Integrating (4.17) over $[0, t]$ in $t$, we obtain (4.9). Next, we estimate $V_{y}$ and $V_{x}$. First, multiplying $\frac{\partial}{\partial y}(4.1)$ by $V_{y}$, we have

$$
\begin{aligned}
\frac{1}{2} \frac{d}{d t}\left\|V_{y}(t)\right\|^{2} & +\int_{R^{2}} V_{y}\{f(U+V)-f(U)\}_{x y} d x d y \\
& +\int_{R^{2}} V_{y} g(U+V)_{y y} d x d y+\mu\left\|\nabla V_{y}(t)\right\|^{2}=0 .
\end{aligned}
$$

The integration by parts gives:

The second and third terms of (4.18)

$$
=\frac{1}{2} \int_{R^{2}} f^{\prime \prime}(U+V) U_{x} V_{y}^{2} d x d y+\frac{1}{2} \int_{R^{2}}\left\{f^{\prime \prime}(U+V) V_{x} V_{y}^{2}+g^{\prime \prime}(U+V) V_{y}^{3}\right\} d x d y .
$$

Hence,

$$
\begin{gathered}
\frac{1}{2} \frac{d}{d t}\left\|V_{y}(t)\right\|^{2}+\frac{\alpha}{2} \int_{R^{2}} U_{x} V_{y}^{2} d x d y+\mu\left\|\nabla V_{y}(t)\right\|^{2} \\
\leq C \int_{R^{2}}\left(\left|V_{y}\right|^{3}+\left|V_{x}\right|\left|V_{y}\right|^{2}\right) d x d y .
\end{gathered}
$$

Since

$$
\begin{aligned}
C & \int_{R^{2}}\left|V_{x} \| V_{y}\right|^{2} d x d y \\
& \leq C \int_{R} \sup _{y \in R}\left|V_{x}(t, x, y)\right|\left\|V_{y}(t, x, \cdot)\right\|_{L^{2}\left(R_{y}\right)}^{2} d x \\
& \leq C \int_{R}\left\|V_{x}(t, x, \cdot)\right\|_{L^{2}\left(R_{y}\right)}^{\frac{1}{2}}\left\|V_{x y}(t, x, \cdot)\right\|_{L^{2}\left(R_{y}\right)}^{\frac{1}{2}}\left\|V_{y}(t, x, \cdot)\right\|_{L^{2}\left(R_{y}\right)}^{2} d x \\
& \leq \frac{\mu}{4}\left\|V_{x y}(t)\right\|_{L^{2}\left(R^{2}\right)}^{2}+C \int_{R}\left\|V_{x}(t, x, \cdot)\right\|_{L^{2}\left(R_{y}\right)}^{\frac{2}{3}}\left\|V_{y}(t, x, \cdot)\right\|_{L^{2}\left(R_{y}\right)}^{\frac{8}{3}} d x \\
\leq & \frac{\mu}{4}\left\|V_{x y}(t)\right\|_{L^{2}\left(R^{2}\right)}^{2} \\
& \quad+C \int_{R}\left\|V_{x}(t, x, \cdot)\right\|_{L^{2}\left(R_{y}\right)}^{\frac{2}{3}}\|V(t, x, \cdot)\|_{L^{2}\left(R_{y}\right)}^{\frac{4}{3}}\left\|V_{y y}(t, x, \cdot)\right\|_{L^{2}\left(R_{y}\right)}^{\frac{4}{3}} d x \\
\leq & \frac{\mu}{4}\left\|\nabla V_{y}(t)\right\|_{L^{2}\left(R^{2}\right)}^{2}+C \int_{R}\left\|V_{x}(t, x, \cdot)\right\|_{L^{2}\left(R_{y}\right)}^{2}\|V(t, x, \cdot)\|_{L^{2}\left(R_{y}\right)}^{4} d x \\
\leq & \frac{\mu}{4}\left\|\nabla V_{y}(t)\right\|_{L^{2}\left(R^{2}\right)}^{2}+C \sup _{x \in R}\|V(t, x, \cdot)\|_{L^{2}\left(R_{y}\right)}^{4}\left\|V_{x}(t)\right\|_{L^{2}\left(R^{2}\right)}^{2} \\
\leq & \frac{\mu}{4}\left\|\nabla V_{y}(t)\right\|_{L^{2}\left(R^{2}\right)}^{2}+C\|V(t)\|_{L^{2}\left(R_{y}\right)}^{2}\left\|V_{x}(t)\right\|_{L^{2}\left(R^{2}\right)}^{4}
\end{aligned}
$$


and

$$
C \int_{R^{2}}\left|V_{y}\right|^{3} d x d y \leq \frac{\mu}{4}\left\|\nabla V_{y}(t)\right\|_{L^{2}\left(R^{2}\right)}^{2}+C\|V(t)\|_{L^{2}\left(R^{2}\right)}^{2}\left\|V_{x}(t)\right\|_{L^{2}\left(R^{2}\right)}^{2}\left\|V_{y}(t)\right\|_{L^{2}\left(R^{2}\right)}^{2},
$$

we have

$$
\begin{aligned}
& \frac{1}{2} \frac{d}{d t}\left\|V_{y}(t)\right\|^{2}+\frac{\alpha}{2} \int_{R^{2}} U_{x} V_{y}^{2} d x d y+\frac{\mu}{2}\left\|\nabla V_{y}(t)\right\|^{2} \\
& \leq C\|V(t)\|_{L^{2}\left(R^{2}\right)}^{2}\left\|V_{x}(t)\right\|_{L^{2}\left(R^{2}\right)}^{2}\left(\left\|V_{x}(t)\right\|_{L^{2}\left(R^{2}\right)}^{2}+\left\|V_{y}(t)\right\|_{L^{2}\left(R^{2}\right)}^{2}\right) .
\end{aligned}
$$

Noting that $\|V(t)\|_{L^{2}\left(R^{2}\right)}^{2} \leq C(1+t)^{-1}$, we multiply $(4.20)$ by $(1+t)^{2+\varepsilon}$ and integrating it over $[0, t]$ to obtain (4.11). Second, multiplying $\frac{\partial}{\partial x}(4.1)$ by $V_{x}$. Then, after similar calculations to the above, we have

$$
\begin{aligned}
\frac{1}{2} \frac{d}{d t}\left\|V_{x}(t)\right\|^{2}+\frac{\alpha}{2} \int_{R^{2}} U_{x}\left|V_{x}\right|^{2} d x d y+\frac{\mu}{2}\left\|\nabla V_{x}(t)\right\|^{2} \\
\leq C\|V(t)\|_{L^{2}\left(R^{2}\right)}^{2}\left\|V_{x}(t)\right\|_{L^{2}\left(R^{2}\right)}^{2}\left(\left\|V_{x}(t)\right\|_{L^{2}\left(R^{2}\right)}^{2}+\left\|V_{y}(t)\right\|_{L^{2}\left(R^{2}\right)}^{2}\right) \\
\quad+C\left\|U_{x}(t)\right\|_{L^{\infty}} \int_{R^{2}} U_{x} V^{2} d x d y .
\end{aligned}
$$

Since $\left\|U_{x}(t)\right\|_{L^{\infty}(R)} \leq\left\|w_{x}(t)\right\|_{L^{\infty}(R)}+\left\|v_{x}(t)\right\|_{L^{\infty}(R)} \leq C(1+t)^{-\frac{7}{8}} \log ^{4}(2+t)$ by virtue of Theorem 5 , we can multiply $(4.21)$ by $(1+t)^{\frac{15}{8}+\varepsilon}$, not $(1+t)^{2+\varepsilon}$, to obtain (4.10). The estimates (4.12)-(4.14) for the second derivatives of $V$ are obtained by more complicated calculations than those for the first derivatives. We omit the details.

Thus the proof of Theorem 7 is complete.

\section{ACKNOWLEDGMENT}

The work of the second author was supported in part by Waseda University Grant for Special Research Project 96A-199.

\section{REFERENCES}

[1] E. Harabetian, Rarefactions and large time behavior for parabolic equations and monotone schemes, Comm. Math. Phys. 114 (1988), 527-536. MR 89d:35084

[2] Y. Hattori and K. Nishihara, A note on the stability of the rarefaction wave of the Burgers equation, Japan J. Indust. Appl. Math. 8 (1991), 85-96. MR 91k:35227

[3] A. M. Il'in and O. A. Oleinik, Asymptotic behavior of solutions of the Cauchy problem for certain quasilinear equations for large time (Russian), Mat. Sb. 51 (1960), 191-216. MR 22:11222

[4] K. Ito, Asymptotic decay toward the planar rarefaction waves of solutions for viscous conservation laws in several space dimensions, Math. Models Methods Appl. Sci. 6 (1996), 315-338. MR 97e:35106

[5] A. Matsumura and K. Nishihara, Asymptotics toward the rarefaction waves of the solutions of a one-dimensional model system for compressible viscous gas, Japan J. Appl. Math. 3 (1986), 1-13. MR 88e:35173

[6] A. Matsumura and K. Nishihara, Global stability of the rarefaction wave of a onedimensional model system for compressible viscous gas, Comm. Math. Phys. 144 (1992), 325-335. MR 93d:76056

[7] A. Matsumura and K. Nishihara, Asymptotics toward the rarefaction wave of the solutions of Burgers' equation with nonlinear degenerate viscosity, Nonlinear Analysis 23 (1994), 605-614. MR 95m:35168

[8] A. Szeppesy and K. Zumbrun, Stability of rarefaction waves in viscous media, Arch. Rational Mech. Anal. 133 (1996), 249-298. MR 97a:35192 
[9] Z. P. Xin, Asymptotic stability of planar rarefaction waves for viscous conservation laws in several dimensions, Trans. Amer. Math. Soc. 319 (1990), 805-820. MR 90j:35138

[10] Z. P. Xin, Asymptotic stability of rarefaction waves for $2 \times 2$ viscous hyperbolic conservation laws-the two-mode case, J. Differential Equations 78 (1989), 191-219. MR 90h:35155

Department of Mathematics, School of Science and Engineering, Waseda University, 3-4-1 Okubo, Shinjuku, Tokyo 169, Japan

E-mail address: masataka@mn.waseda.ac.jp

School of Political Science and Economics, Waseda University Tokyo, 169-50, Japan

E-mail address: kenji@mn.waseda.ac.jp 